\title{
Midlife alcohol consumption and longitudinal brain atrophy: the PREVENT-Dementia study
}

\author{
Michael J. Firbank ${ }^{1}$ (D) John T. O'Brien ${ }^{2} \cdot$ Karen Ritchie $^{3,4} \cdot$ Katie Wells $^{5} \cdot$ Guy Williams $^{6} \cdot$ Li Su $^{2} \cdot$ Craig W. Ritchie $^{4}$
}

Received: 24 April 2020 / Revised: 11 June 2020 / Accepted: 12 June 2020 / Published online: 20 June 2020

(c) The Author(s) 2020

\begin{abstract}
Background and aims Consensus is lacking on whether light to moderate consumption of alcohol compared to abstinence is neuroprotective. In this study, we investigated the relationship between self-reported alcohol use and brain volume change over 2 years in middle-aged subjects.

Methods A sample of 162 subjects (aged 40-59 at baseline) from the PREVENT-Dementia programme underwent MRI scans on two separate occasions (mean interval 734 days; SD 42 days). We measured longitudinal rates of brain atrophy using the FSL Siena toolbox, and change in hippocampal volume from segmentation in SPM.

Results Controlling for age and sex, there were no significant associations of either total brain, ventricular, or hippocampal volume change with alcohol consumption. Adjusting for lifestyle, demographic and vascular risk factors did not alter this. Conclusions We did not find any evidence of influence of alcohol consumption on changes in brain volume over a 2-year period in 40-60-year-olds.
\end{abstract}

Keywords MRI $\cdot$ Alcohol $\cdot$ Mid-life $\cdot$ Brain volume $\cdot$ Longitudinal $\cdot$ Atrophy

\section{Introduction}

High consumption of alcohol has consistently been linked with dementia and brain degeneration; however, several, but not all, studies have suggested that moderate consumption vs. abstinence is a protective factor against dementia [1, 2].

Michael J. Firbank

michael.firbank@ncl.ac.uk

1 Institute of Neuroscience, Newcastle University, Campus for Ageing and Vitality, Nuns Moor Road, Newcastle upon Tyne NE4 5PL, UK

2 Department of Psychiatry, University of Cambridge, Level E4 Cambridge Biomedical Campus, Box 189, Cambridge CB2 OSP, UK

3 INSERM, Montpellier, France

4 Centre for Dementia Prevention, University of Edinburgh, Edinburgh, UK

5 The Centre for Psychiatry, Imperial College London, 7th floor Commonwealth Building, Du Cane Road, London W12 0NN, UK

6 Department of Clinical Neurosciences and Wolfson Brain Imaging Centre, University of Cambridge, Cambridge Biomedical Campus, Cambridge CB2 0SP, UK
Some studies of the association of alcohol consumption with brain volume have similarly found protective effects of light to moderate drinking [3], whilst others have found that brain volume tends to linearly decrease with increased alcohol intake [4]. Topiwala et al. [5] found higher alcohol consumption associated with smaller hippocampi, as contrasted with Downer et al. [6] who found larger hippocampi for those with moderate consumption. A systematic review concluded that hippocampal volume was reduced in those with problematic alcohol use [7]. All these studies used cross-sectional imaging data, and are thus not sensitive to any ongoing neurodegeneration.

The aim of this study was to investigate alcohol consumption and longitudinal changes in brain volume in middleaged adults. We investigated whole brain and ventricular volume as well as hippocampal volume, due to its association with both alcohol dependence and dementia. We hypothesised that increased alcohol consumption would be associated with brain atrophy. 


\section{Materials and methods}

\section{Subjects}

The protocol has been described in detail elsewhere [8]. A total of 193 participants aged 40-59, of whom 168 had repeat MRI were recruited through multiple sources. Initially they were identified from the dementia register database held at a London National Health Service (NHS) Trust, part of the UK National Health Service. Other participants were recruited via the Join Dementia Research website (https ://www.joindementiaresearch.nihr.ac.uk/), through information about the study on the Internet and public presentations. The study aimed to recruit approximately half of the subjects with a parental family history of dementia and half without. All MRI scans obtained on participants were formally reported by an experienced consultant neuroradiologist. Any subject with significant incidental findings (e.g., tumour, stroke) was excluded. Approval for the study was given by the NHS Research Ethics Committee London Camberwell St-Giles (REC reference: 12/LO/1023). All participants provided informed written consent.

\section{Imaging}

Participants underwent multimodal $3 \mathrm{~T}$ structural magnetic resonance imaging on a single scanner (Siemens Verio) including volumetric T1-weighted scans (176 slices, $1.0 \times 1.0 \mathrm{~mm}, 1.0 \mathrm{~mm}$ slice thickness, repetition time $=2300 \mathrm{~ms}$, echo time $=2.98 \mathrm{~ms}$, flip angle $9^{\circ}$ ). Scans were repeated after approximately 2 years on the same scanner using the same protocol. Imaging findings from these participants have been previously reported [9-11].

Percentage brain and ventricular volume change between the two scans were determined using the FSL SIENA program (https://fsl.fmrib.ox.ac.uk/fsl/fslwiki). Values were then divided by the time between scans to give rates per year. SPM12 (https://www.fil.ion.ucl.ac.uk/ spm) was used to segment the brain into grey matter, white matter and CSF, and spatially normalise the images to standard space. To determine hippocampal volume, the hippocampal region of interest (ROI) from the AAL template [12] was used to determine the mean of the normalised grey matter segmentation image. Left and right were averaged, and the longitudinal change was determined by subtracting the baseline measurement from repeat. Total intracranial volume (ICV) was calculated from the sum of grey matter, white matter and CSF segmentation at baseline. All output was manually checked to ensure correct brain segmentation, and alignment of baseline/repeat scans.

\section{Lifestyle variables}

Demographic data and information on drinking and smoking status were collected from case report forms which hold participants' data collected at interview. We also included data from the participants' medical history, which included history of alcoholism and diabetes. Participants were classified as current smokers or non-smokers (never and former).

We used the Lifetime of Experiences Questionnaire (LEQ) [13] to extract data about physical, social and cultural activity (art, music, hobbies) from the age of 30 years to present. The three physical questions used from the LEQ asked participants how often they engaged in activities that were (1) mildly energetic (such as walking), (2) moderately energetic and (3) vigorous (such as running). For each activity, participants were asked to indicate their response on the six-point Likert scale from Never to Daily. We dichotomised the scores as to whether people did the activities more vs. less frequently than once a month.

Alcohol consumption in units per week was determined using the Scottish Collaborative Group Food Frequency Questionnaire version 7.1 (https://www.foodfrequency. org) completed by the participant at baseline and repeat visit. The questionnaire asks for the typical amount and frequency of consumption of 175 food and drink items over the last 2-3 months. We took the average of baseline and repeat alcohol consumption, except for eight participants for whom repeat values were missing, in which case we used the baseline value. Following previous research [5] and the UK guidelines of 14 units per week as a safe limit (https://www.drinkaware.co.uk/alcoholfacts/alcoholic-drinks-units/latest-uk-alcohol-unit-guida nce/), alcohol usage was divided into abstaining ( $<1$ unit/ week), light ( $>=1$ and $<7$ units/week), moderate $(>=7$ and $<14$ units/week), high $(>=14$ and $<21$ units/week) and very high ( $>=21$ units per week). No distinction was applied between men and women in this classification. For a subgroup analysis on male and female separately, we divided alcohol use into three groups: (0-5), (>5-15), ( $>15)$ units per week to avoid having too few male subjects in each group.

The Framingham risk score was used to calculate 10-year cardiovascular risk from current smoking status, systolic BP, BP medication status, sex, age at baseline, total and HDL cholesterol and diagnosis of diabetes. Social class was determined using the UK National Statistics Socio-economic classification (NS-SEC). 


\section{Statistical analysis}

Brain volume data and clinical variables were analysed with $\mathrm{R}$ version 3.5.2. Independent $t$ tests were used to compare groups for continuous variables. Linear regression was used to investigate association of alcohol with brain atrophy rates. Total brain and ventricular volume change were expressed as percentage of baseline volume (as calculated by SIENA). For hippocampal analysis, we included ICV as a covariate to adjust for head size. Residuals were checked by eye to verify normality, heteroscedasticity and absence of nonlinear associations.

\section{Results}

Of the 193 participants with T1 MR scans at baseline, 168 had a repeat scan. One participant was excluded as an outlier (ventricular change was negative, 3SD below mean). We excluded one subject due to missing alcohol consumption data, and four with a history of alcohol dependence, leaving 162 in the main analysis.

Mean age at baseline was 52 year (SD 5.4), and mean interval between scans 734 (SD 42) days. There were 24 alcohol abstainers, 42 light, 44 moderate, 20 high and 32 very high consumers of alcohol. The highest intake group included six persons (four male) drinking $>50$ units/week. There were 112 female and 50 male participants, with men having higher alcohol consumption than women 19.7 (SD 21.3 ) vs. 10.9 (SD 12.1) units/week $t_{63.5}=2.74, p=0.008$, Welch two-sample $t$ test.

Mean Framingham risk score was 6.6\% (SD 5.5\%, $\min =0.9 \%, \max =47 \%$ ). There were $25 / 162$ participants who engaged in artistic, musical activities or hobbies at least once a fortnight; 149/161 who met family or friends; $151 / 162$ engaged in mild, 128/162 moderate, and 76/162 vigorous activities. 85 participants had one or both parents with dementia, and there were six current smokers. Mean years of education was 16.1 (SD 3.4). There were 36 people in social class one, 56 in class two, 22 in class three, 10 in class four, 7 in classes five-seven and 26 in class eight, with 5 people not classified. 35 people had a medical history of depression, two of whom had a recurrence during the study, and a further two had incident depression. Supplementary Tables 1 and 2 present these data split by alcohol consumption. The moderate consumption group had the lowest Framingham score, which was significantly lower than the abstainer group ( $p=0.02$, Tukey HSD post hoc). The abstainer group had significantly fewer years of education than the moderate $(p=0.007$ Tukey HSD post hoc) and the very high group ( $p=0.03$ Tukey HSD post hoc).

In linear models including alcohol consumption, age, sex (and intracranial volume for hippocampus), there was no significant effect of alcohol on brain change (Table 1), although there was a trend $(p=0.09)$ of association between drinking 14-21 units of alcohol and preserved total brain volume (Table 1, Fig. 1). Addition of other factors (education, midlife activities, social class, vascular risk factors, family history of dementia) did not alter this lack of significant association between alcohol and longitudinal brain change [supplementary Tables 3,4]. The addition of the four subjects with a history of alcohol dependence did not qualitatively alter the results. As sex was a strong predictor of atrophy, we also repeated the linear model analysis in male and female participants separately. In this, we split alcohol usage into three groups due to relatively low numbers of male participants. The demographics and results of the linear model are in Supplementary Tables 5-7. There were no significant associations of brain volume change with alcohol in this analysis.

Table 1 Linear model predictors of annual percentage ventricle change, total brain volume change and hippocampal volume change

\begin{tabular}{|c|c|c|c|c|c|c|}
\hline & \multicolumn{2}{|c|}{$\begin{array}{l}\text { Percent ventricle change per year } \\
\mathrm{F}_{6,155}=6.4 ; p<0.001\end{array}$} & \multicolumn{2}{|c|}{$\begin{array}{l}\text { Percent brain volume change per year } \\
\mathrm{F}_{6,155}=2.98 ; p<0.009\end{array}$} & \multicolumn{2}{|c|}{$\begin{array}{l}\text { Hippocampal volume change per year } \\
\mathrm{F}_{7,154}=3.58 ; p=0.001\end{array}$} \\
\hline & Beta (SE) & & Beta (SE) & & Beta (SE) & \\
\hline Age & $0.16(0.04)$ & $t=4.59 ; p=<0.001 * *$ & $-0.017(0.005)$ & $\begin{aligned} t= & -3.68 \\
p & =<0.001 * *\end{aligned}$ & $-0.11(0.60)$ & $t=-0.19 ; p=0.848$ \\
\hline Male sex & $1.60(0.43)$ & $t=3.75 ; p=<0.001 * *$ & $0.042(0.056)$ & $t=0.75 ; p=0.452$ & $0.70(9.77)$ & $t=0.07 ; p=0.943$ \\
\hline 1-7 units/week & $0.51(0.62)$ & $t=0.82 ; p=0.414$ & $0.073(0.081)$ & $t=0.90 ; p=0.368$ & $10.68(10.33)$ & $t=1.03 ; p=0.303$ \\
\hline 7-14 units/week & $0.72(0.62)$ & $t=1.16 ; p=0.247$ & $0.055(0.081)$ & $t=0.69 ; p=0.493$ & $0.09(10.48)$ & $t=0.01 ; p=0.993$ \\
\hline 14-21 units/week & $-0.49(0.73)$ & $t=-0.67 ; p=0.502$ & $0.162(0.095)$ & $t=1.70 ; p=0.091$ & $2.73(12.37)$ & $t=0.22 ; p=0.826$ \\
\hline$>21$ units/week & $0.10(0.66)$ & $t=0.15 ; p=0.883$ & $0.061(0.085)$ & $t=0.71 ; p=0.478$ & $9.52(10.96)$ & $t=0.87 ; p=0.386$ \\
\hline Intracranial volume & & & & & $-118.5(35.0)$ & $t=-3.39 ; p=<0.001^{* *}$ \\
\hline
\end{tabular}

Alcohol values are referenced to the abstainer group $(<1$ unit alcohol per week)

$* * p<0.001$ 


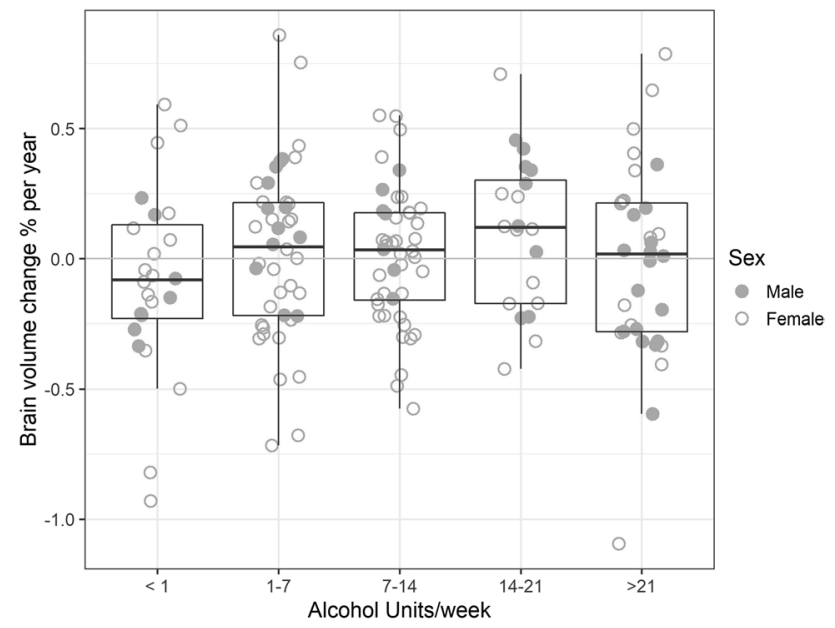

Fig. 1 Percentage brain volume change per year for different alcohol intake groups. Negative values indicate brain shrinkage

\section{Discussion}

Contrary to our hypothesis, we did not observe any significant association between alcohol consumption and longitudinal brain volume changes. Rather, we saw a non-significant trend of 14-21 units of alcohol (vs. abstinence) associated with preserved total brain volume.

Studies of alcohol on cognition or brain structure have confounds due to social and demographic factors, with age, years of education, and social class all being linked to both alcohol consumption and brain volume. Our study, looking at brain volume change over 2 years within individuals overcomes to some extent these confounds, and including the lifestyle, demographic and vascular health factors in our analysis did not change our findings.

Combined with the previous conflicting reports on the benefit or otherwise of mild to moderate alcohol consumption, our data suggest at least that consumption of 7-21 units per week is not associated with marked brain atrophy over a 2-year period in midlife.

Limitations of the study include that alcohol consumption was estimated from subject report, and the relatively short follow-up of 2 years. The participants were mostly female, limiting the extrapolation to the general population.

In summary, we did not find any evidence of influence of alcohol consumption on changes in brain volume over a 2 -year period in 40-60-year-olds.

Acknowledgements This work was funded by a Grant for the PREVENT-Dementia programme from the UK Alzheimer's Society (Grant numbers 178 and 264), and the PREVENT Dementia study is also supported by the US Alzheimer's Association (Grant number TriBEKa-17-519007), and philanthropic donations. JOB and LS are supported by the Cambridge NIHR Biomedical Research Centre. MJF is supported by the NIHR Newcastle Biomedical Research Centre awarded to the Newcastle Hospitals NHS Foundation Trust and Newcastle University. LS is also supported by Alzheimer's Research UK (ARUK-SRF2017B-1). Participants were recruited at West London Mental Health National Health Service (NHS) Trust (now known as West London NHS Trust) and scanning was carried out at the Clinical Imaging Facility, Imperial College London. We thank all the PREVENT-Dementia participants for their enthusiastic participation in this study.

Author contributions MJF performed the image analysis, statistical analysis and wrote the paper. JTO'B helped obtain funding, led the design of the imaging protocol and supervised this study. KR helped obtain funding and was involved with study design. KW was involved with study design and oversaw data collection. GW assisted with the detailed design and implementation of the imaging protocol. CWR helped obtain funding, was involved with study design and is the Chief Investigator of the PREVENT-Dementia programme. LS assisted with the detailed design and implementation of the imaging protocol and supervised all aspects of the MR data design and collection.

Funding This work was funded by a Grant for the PREVENT-Dementia programme from the UK Alzheimer's Society (Grant numbers 178 and 264), and the PREVENT Dementia study is also supported by the US Alzheimer's Association (Grant number TriBEKa-17-519007), and philanthropic donations.

\section{Compliance with ethical standards}

Conflicts of interest Michael J. Firbank has no conflicts of interest to declare. John T. O'Brien has no conflicts related to this study. Unrelated to this work he has received honoraria for work as DSMB chair or member for TauRx, Axon, Eisai, has acted as a consultant for Lilly and Roche, and has received honorarium for talks from GE Healthcare and research support from Alliance Medical. Karen Ritchie, Katie Wells, Guy Williams, Craig W. Ritchie and Li Su have no conflicts of interest to declare.

Ethical approval Approval for the study was given by the NHS Research Ethics Committee London Camberwell St-Giles (REC reference: 12/LO/1023). All participants provided informed written consent.

Open Access This article is licensed under a Creative Commons Attribution 4.0 International License, which permits use, sharing, adaptation, distribution and reproduction in any medium or format, as long as you give appropriate credit to the original author(s) and the source, provide a link to the Creative Commons licence, and indicate if changes were made. The images or other third party material in this article are included in the article's Creative Commons licence, unless indicated otherwise in a credit line to the material. If material is not included in the article's Creative Commons licence and your intended use is not permitted by statutory regulation or exceeds the permitted use, you will need to obtain permission directly from the copyright holder. To view a copy of this licence, visit http://creativecommons.org/licenses/by/4.0/.

\section{References}

1. Handing EP, Andel R, Kadlecova P, Gatz M, Pedersen NL (2015) Midlife alcohol consumption and risk of dementia over 43 years of follow-up: a population based study from the Swedish twin 
registry. J Gerontol Series A Biol Sci Med Sci 70:1248-1254. https://doi.org/10.1093/gerona/glv038

2. Ruitenberg A, van Swieten JC, Witteman JC, Mehta KM, van Duijn CM, Hofman A, Breteler MM (2002) Alcohol consumption and risk of dementia: the Rotterdam study. Lancet 359:281-286. https://doi.org/10.1016/S0140-6736(02)07493-7

3. Gu Y, Scarmeas N, Short EE, Luchsinger JA, DeCarli C, Stern Y, Manly JJ et al (2014) Alcohol intake and brain structure in a multiethnic elderly cohort. Clin Nutr 33:662-667. https://doi. org/10.1016/j.clnu.2013.08.004

4. Paul CA, Au R, Fredman L, Massaro JM, Seshadri S, DeCarli C, Wolf PA (2008) Association of alcohol consumption with brain volume in the Framingham study. JAMA Neurol 65:1363-1367. https://doi.org/10.1001/archneur.65.10.1363

5. Topiwala A, Allen CL, Valkanova V, Zsoldos E, Filippini N, Sexton C, Mahmood A et al (2017) Moderate alcohol consumption as risk factor for adverse brain outcomes and cognitive decline: longitudinal cohort study. BMJ 357:j2353. https://doi.org/10.1136/ bmj.j2353

6. Downer B, Jiang Y, Zanjani F, Fardo D (2015) Effects of alcohol consumption on cognition and regional brain volumes among older adults. Am J Alzheimers Dis Other Demen 30:364-374. https://doi.org/10.1177/1533317514549411

7. Wilson S, Bair JL, Thomas KM, Iacono WG (2017) Problematic alcohol use and reduced hippocampal volume: a meta-analytic review. Psychol Med 47:2288-2301. https://doi.org/10.1017/ S0033291717000721

8. Ritchie CW, Ritchie K (2012) The PREVENT study: a prospective cohort study to identify mid-life biomarkers of late-onset Alzheimer's disease. BMJ Open 2:e01893. https:// doi.org/10.1136/bmjopen-2012-001893

9. Ritchie K, Carrière I, Su L, O'Brien JT, Lovestone S, Wells K, Ritchie CW (2017) The midlife cognitive profiles of adults at high risk of late-onset Alzheimer's disease: the PREVENT study. Alzheimers Dement 13:1089-1097. https://doi.org/10.1016/j. jalz.2017.02.008

10. Stefaniak JD, Su L, Mak E, Sheikh-Bahaei N, Wells K, Ritchie $\mathrm{K}$, Waldman A et al (2018) Cerebral small vessel disease in middle age and genetic predisposition to late-onset Alzheimer's disease. Alzheimers Dement 14:253-258. https://doi.org/10.1016/j. jalz.2017.08.017

11. O’Brien JT, Firbank MJ, Ritchie K, Wells K, Williams GB, Ritchie CW, Su L (2020) Association between midlife dementia risk factors and longitudinal brain atrophy: the PREVENT-dementia study. J Neurol Neurosurg Psychiatry 91:158-161. https://doi. org/10.1136/jnnp-2019-321652

12. Tzourio-Mazoyer N, Landeau B, Papathanassiou D, Crivello F, Etard O, Delcroix N, Mazoyer B et al (2002) Automated anatomical labeling of activations in SPM using a macroscopic anatomical parcellation of the MNI MRI single-subject brain. NeuroImage 15:273-289. https://doi.org/10.1006/nimg.2001.0978

13. Valenzuela MJ, Sachdev P (2007) Assessment of complex mental activity across the lifespan: development of the lifetime of experiences questionnaire (LEQ). Psychol Med 37:1015-1025. https:// doi.org/10.1017/S003329170600938X 